\title{
Optical Fiber Sensor Experimental Research Based on the Theory of Bending Loss Applied to Monitoring Differential Settlement at the Earth-Rock Junction
}

\author{
Jianchun Qiu, ${ }^{1,2,3}$ Dongjian Zheng, ${ }^{1,2,3}$ Kai Zhu, ${ }^{2,3}$ Bin Fang, ${ }^{4}$ and Lin Cheng ${ }^{5}$ \\ ${ }^{1}$ State Key Laboratory of Hydrology-Water Resources and Hydraulic Engineering, Hohai University, Nanjing 210098, China \\ ${ }^{2}$ National Engineering Research Center of Water Resources Efficient Utilization and Engineering Safety, Hohai University, \\ Nanjing 210098, China \\ ${ }^{3}$ College of Water-Conservancy and Hydropower, Hohai University, Nanjing 210098, China \\ ${ }^{4}$ HYDROCHINA Guiyang Engineering Corporation Co., Ltd., Guiyang 550081, China \\ ${ }^{5}$ State Key Laboratory Base of Eco-Hydraulic Engineering in Arid Area, Xian University of Technology, Xian 710048, China \\ Correspondence should be addressed to Jianchun Qiu; 121267184@qq.com
}

Received 12 April 2015; Revised 5 June 2015; Accepted 8 June 2015

Academic Editor: Kourosh Kalantar-Zadeh

Copyright ( 2015 Jianchun Qiu et al. This is an open access article distributed under the Creative Commons Attribution License, which permits unrestricted use, distribution, and reproduction in any medium, provided the original work is properly cited.

Considering the differential settlement in the junction between the structure perpendicular to the dike and the body and foundation of dike (called the earth-rock junction in this paper) during runtime, an experimental investigation of optical fiber sensor monitoring was conducted. Based on the sensing mechanism of single-mode optical fiber bending loss, the experiment focused on the influence of the bending radius of an optical fiber on the bending loss. In view of the characteristics of the differential settlement in the earth-rock junction, we designed a butterfly-type optical fiber sensor and composite optical fiber sensor for monitoring device in monitoring the differential settlement to enlarge the monitoring range and improve the sensibility of optical fiber sensor. Based on the research on the working principle and bending properties of the composite optical fiber monitoring device, we conducted experiments on the bending of the composite optical fiber sensor monitoring device and the use of the device for monitoring the differential settlement. These experiments verified the feasibility of the composite optical fiber sensor monitoring device at monitoring the differential settlement in the earth-rock junction.

\section{Introduction}

Dike projects have played important roles in increasing the social benefits of flood control, irrigation, water supply, and navigation. The safety of a dike project is closely related to people's livelihoods. Because distinct disparities exist in the properties and structure of two materials in the contact region between the building across the dike and the body and foundation of dike, there is a high possibility of differential settlement in the earth-rock junction. Therefore, it is important to conduct safety monitoring for the junction to determine any potential safety hazards and maintain the overall safety of the dike.

The current, monitoring equipment for structures across dikes includes a clinometer, settlement gauge, vibration string joint meter, and vibration string displacement meter. However, such monitoring equipment has the disadvantages of high unit cost, poor durability, low accuracy, easy interferences under high temperature and electromagnetism, and failure to recognize distributed monitoring. However, with developments in science and technology, especially the development of optical technology, monitoring methods based on optical fiber sensors have become an important means of safety monitoring. An optical fiber sensor can provide sensing and transmission monitoring and is capable of functioning remotely. It also boasts the features of being light in weight and small in volume, having less effect on the structure. It has the ability to monitor under adverse environments, such as high temperature and high electromagnetism. Once connected with a computer, the monitoring system can be 
automated and intelligent, capable of distanced monitoring the structural damage.

By far, optical fiber sensors have made major achievements in dams [1-4], tunnels $[5,6]$, slopes $[7,8]$, and bridges $[9,10]$. However, the research into optical fiber sensors applied to dikes is limited. Focusing on the differential settlement of the earth-rock junction, we investigated the application of optical fiber sensors to monitoring dikes by comprehensively implementing the theoretical analysis and model test to provide technical support for mastering the security of the earth-rock junction.

\section{Transmission Characteristics of Optical Fibers}

2.1. Structure and Transmission Characteristics of Optical Fibers. According to the propagation law of light rays, reflection and refraction often appear simultaneously when light ray flows from one medium to another. When light ray flows from an optically thinner medium to an optically denser medium, the refraction angle is less than incident angle, while the incident angle is less than the refraction angle when light ray flows from an optically denser medium to an optically thinner medium. When the incident angle reaches a certain value, refraction does not happen and light ray would be reflected completely. Therefore, the condition of total reflection is described by the following:

$$
\begin{gathered}
n_{1}>n_{2}, \\
\alpha>\theta,
\end{gathered}
$$

where $n_{1}$ is the refractive index of optically denser medium, $n_{2}$ is the refractive index of optically thinner medium, $\alpha$ is the incident angle, $\theta$ is the critical angle, and $\theta=\arcsin \left(n_{2} / n_{1}\right)$.

Since light ray waves are propagated in an optical fiber through total reflection, an optical fiber is often designed to have a fiber core, cladding, and coating. The profile structure of an optical fiber is shown in Figure 1. To have a larger refractive index for the fiber core, the main material of the fiber core is silicon dioxide $\left(\mathrm{SiO}_{2}\right)$ doped with germanium dioxide $\left(\mathrm{GeO}_{2}\right)$, and the diameter of the optical fiber is 5$50 \mu \mathrm{m}$. However, the refractive index of the cladding is just less than that of the fiber core, with the main material being silicon dioxide, and the normal diameter of the cladding is often $125 \mu \mathrm{m}$. The main material of the coating is a high polymer material, such as epoxy resin or silicone rubber, and its diameter is approximately $250 \mu \mathrm{m}$. An optical fiber has increased mechanical strength and flexibility due to its coating.

\subsection{Light Ray Transmission Characteristics of Curved Optical} Fibers. Although light rays in an optical fiber follow the principle of total reflection, the light ray intensity might be weakened if the light ray flows from one end of the optical fiber to the other, which indicates that there is a loss for light rays during the propagation process. When an optical fiber is bent to a certain degree, the propagation path of the light rays

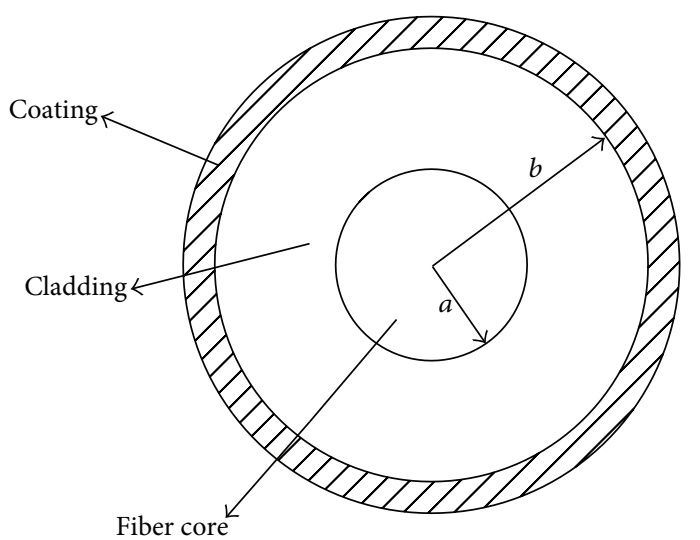

FIgURE 1: The profile structure of optical fiber.

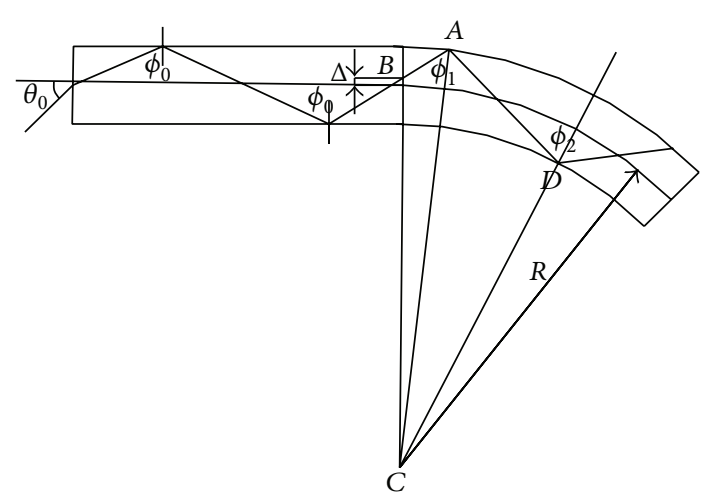

FIGURE 2: Light ray transmission in the meridian plane of the curved optical fiber.

would change and a portion of the light ray would infiltrate into the cladding or even pass through the cladding.

In this work, we employed geometrical optics to analyze the transmission characteristics of light rays in the case of curved optical fibers. In fact, the optical fibers in engineering detection are often curved. Therefore, the light ray propagation of optical fibers does not conform to the total reflection of meridional light ray (any plane passing through the central axis of an optical fiber is defined as a meridian plane, and the light ray inside of the meridian plane is defined as a meridional light ray).

Figure 2 shows the light ray transmission in the meridian plane of a curved optical fiber. The bending radius of the curved optical fiber is $R$, and the radius of fiber core is $a$. We only analyzed the propagation of light in the meridian plane, and the incident angles in meridian plane are $\phi_{0}, \phi_{1}$, and $\phi_{2}$. If the incident angle of $\phi_{1}$ is less than that of $\phi_{2}$, the reflected light in the upper part would overflow the coating.

Point $B$ indicates the initial bending point of the optical fiber, and Point $C$ is the center of the bending arc. For the triangle $A B C$, according to the sine theorem, we can conclude the following:

$$
\sin \phi_{1}=\frac{C B}{C A} \sin \left(\pi-\phi_{0}\right)=\frac{R+\Delta}{R+a} \sin \phi_{0}
$$


where $\Delta$ is the distance between Point $B$ and the axis of the optical fiber.

Clearly, for the triangle $A B C$, the following relationship holds true:

$$
C B \leq C A \text {. }
$$

Hence, we can conclude the following:

$$
\sin \phi_{1} \leq \sin \phi_{0}, \quad \phi_{1} \leq \phi_{0} .
$$

Thus, a partition of light would overflow from Point $A$.

Similarly, for the triangle $A C D$, the following equation can be obtained:

$$
\begin{aligned}
\sin \phi_{2} & =\frac{C A}{C D} \sin \phi_{1}=\frac{C A}{C D} \frac{C B}{C A} \sin \phi_{0}=\frac{C B}{C D} \sin \phi_{0} \\
& =\frac{R+\Delta}{R-a} \sin \phi_{0} .
\end{aligned}
$$

Then,

$$
\sin \phi_{2} \geq \sin \phi_{0}, \quad \phi_{2} \geq \phi_{0} .
$$

Therefore, light would not overflow the concave.

According to the geometrical relationship in Figure 2, the following equation is obtained:

$$
S_{0}=\frac{\sin a}{a}\left(1-\frac{a}{R}\right) S_{c}
$$

where $R$ is the curvature radius, $S_{0}$ is the optical path length of the meridional light ray in unit lengths of the curved optical fiber, and $S_{c}$ is the optical path length of meridional light ray in unit lengths of a straight optical fiber.

Clearly, we can determine the following relationship:

$$
\begin{aligned}
\frac{\sin a}{a} & <1, \\
0 & <1-\frac{a}{R}<1 .
\end{aligned}
$$

Then,

$$
S_{0}<S_{c} \text {. }
$$

In equation (9) illustrates that the optical path length of the meridional light ray in unit lengths of the curved optical fiber is shorter. This relationship demonstrates that the amount of reflection in unit lengths of the curved optical fiber is less than that of a straight optical fiber.

\section{Bending Loss Mechanism of Optical Fiber Sensing}

The bending loss of an optical fiber includes macrobending loss and microbending loss. Macrobending loss dominates when the curvature radius of the optical fiber is much larger than the diameter of the optical fiber; meanwhile, microbending loss occurs when the curvature radius and the diameter of optical fiber are of the same order of magnitude. Because the optical fiber is based on the theory of bending loss in monitoring the differential settlement in the earthrock junction, a large monitoring range and high sensibility are required. Therefore, both the microbending loss and macrobending loss are included.
3.1. Microbending Loss Mechanism of Optical Fiber. Due to the changes in temperature or uploading, the axis of an optical fiber has bending deflections at the micron level, and the induced loss is called microbending loss. Certainly, the bending deflection influences the light ray intensity, as a partition of light ray would enter or overflow the cladding. Generally, the microbending loss of the optical fiber demodulates via the microbending sensor. As a type of intensity modulated sensor, an optical fiber microbending sensor could acquire the variation of external parameters by measuring the variation of optical power of the fiber core or cladding.

The magnitude of microbending loss is described by the following:

$$
A_{m}=N\left\langle h^{2}\right\rangle \frac{a^{4}}{c^{6} \Delta_{2}^{3}}\left[\frac{E}{E_{f}}\right]^{3 / 2},
$$

where $N$ is the number of microbending section, $h$ is the projecting height of the bending, $\left\langle h^{2}\right\rangle$ indicates the average of $h^{2}, a$ is the radius of fiber core, $c$ is the external diameter of the optical fiber, $E$ is the elastic modulus of the coating, $E_{f}$ is the elastic modulus of the fiber core, and $\Delta_{2}$ is the difference between the refractive index of the fiber core and cladding.

By analyzing (10), we find that the magnitude of microbending loss $A_{m}$ is proportional to the fourth power of the radius of the fiber core $a$ and inversely proportional to the relative difference of the refractive index $\Delta_{2}$. Hence, to increase the micro-bending loss, the radius of the fiber core $a$ should be increased or the external diameter of the optical fiber $c$ and the relative difference of the refractive index $\Delta_{2}$ should be decreased.

3.2. Macrobending Loss Mechanism of Optical Fibers. According to the principle of fiber optics [11], when the curvature radius $R$ is larger than the critical value $R_{c}\left(R>R_{c}\right)$, the induced bending loss is too slight to be neglected. The bending loss increases exponentially when the curvature radius $R$ is smaller than the critical value $R_{c}\left(R>R_{c}\right)$. Therefore, the critical value $R_{c}$ is of great importance for the design, studies, and applications of optical fibers.

The expression of macrobending loss is given as follows:

$$
a_{c}=A_{c} R^{-1 / 2} \exp (-U R) \text {. }
$$

The parameter $A_{c}$ and the critical value of $U$ are given as follows:

$$
\begin{aligned}
& A_{c} \approx 30\left(\Delta_{3}\right)^{1 / 4} \lambda^{-1 / 2}\left(\frac{\lambda_{c}}{\lambda}\right)^{3 / 2} R \exp (-U R), \\
& U_{c} \approx 0.705 \frac{(\Delta)^{3 / 2}}{\lambda}\left(2.748-0.996 \frac{\lambda}{\lambda_{c}}\right)^{3},
\end{aligned}
$$

where $\Delta_{3}$ is the relative difference of refractive index, $\lambda$ is the wavelength, and $\lambda_{c}$ is the cutoff wavelength.

By substituting (12) into (11), the critical radius $R_{c}$ is obtained:

$$
R_{c} \approx 20 \frac{\lambda}{(\Delta)^{3 / 2}}\left(2.748-0.996 \frac{\lambda}{\lambda_{c}}\right)^{-3} .
$$




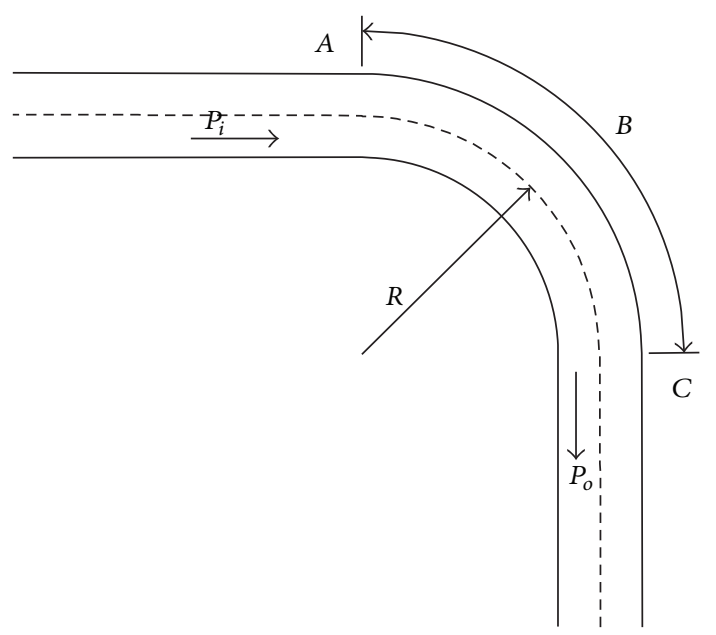

FIGURE 3: The diagram of macrobending loss of optical fiber.

The maximum and minimum cutoff wavelengths of the single-mode optical fiber used in our experiments are $\lambda_{c 1}=$ $1144 \mathrm{~nm}, \lambda_{c 2}=1187 \mathrm{~nm}$, respectively, and the difference in refractive index between the fiber core and cladding $(\Delta)$ is 0.0015 .

Macrobending loss includes the pure bending loss and the transitional bending loss. Figure 3 shows the macrobending loss diagram of the optical fiber; the pure bending is located at the position around $A$ and $C$, while the transitional bending loss is located between $A$ and $C$.

As Figure 3 shows, the initial power of the optical fiber is $P_{i}$, and the output power is $P_{o}$. The following equation is obtained after neglecting the inherent attenuation of the optical fiber:

$$
\frac{P_{o}}{P_{i}}=\left.\left.\left.\frac{P_{o}}{P_{i}}\right|_{A} \frac{P_{o}}{P_{i}}\right|_{B} \frac{P_{o}}{P_{i}}\right|_{C},
$$

where the positions of $A$ and $C$ correspond to the bending transition loss and the position of $B$ corresponds to the pure bending loss.

For the symmetric structure, the following equation is given:

$$
\left.\frac{P_{o}}{P_{i}}\right|_{A}=\left.\frac{P_{o}}{P_{i}}\right|_{C} .
$$

For the section of $B$, the following equation is given:

$$
\left.\frac{P_{o}}{P_{i}}\right|_{B}=\exp (-2 \alpha L),
$$

where $\alpha$ is the coefficient of bending loss of the optical fiber and $L$ is the bending length of optical fiber.

By substituting (15) and (16) into (14) and taking the logarithm of (14), we obtain the following equation:

$$
\ln \left(\frac{P_{o}}{P_{i}}\right)=-2 \alpha L+2 \ln \left(\left.\frac{P_{o}}{P_{i}}\right|_{A}\right) .
$$

According to the research by Zhu [12], for a certain wavelength, a linear relationship exists between the bending loss and bending angle. The intercept of $\ln \left(P_{o} / P_{i}\right)$ on the vertical coordinate is the transitional bending loss, which is a fixed value and shares a small proportion. Therefore, the pure bending loss is the main part of the bending loss, which can be treated approximately as the bending loss for simplicity. Then, the following equation is obtained:

$$
\frac{P_{o}}{P_{i}}=\left.\frac{P_{o}}{P_{i}}\right|_{B}=\exp (-2 \alpha L) \text {. }
$$

In practical engineering applications, the bending loss of the optical fiber is often given in decibels $(\mathrm{dB})$, and the power attenuation decibels of the optical fiber with a length of $L$ is described by the following:

$$
\begin{aligned}
L_{s} & =10 \log _{10}\left(\frac{P_{i}}{P_{o}}\right)=10 \log _{10}\left[\frac{1}{\exp (-2 \alpha L)}\right] \\
& =4.342(2 \alpha L) .
\end{aligned}
$$

Marcuse [13] provided the expression for the bending loss coefficient of a single-mode optical fiber under the weakguiding conditions:

$$
2 \alpha=\frac{\sqrt{\pi} \kappa^{2}}{2 \gamma^{3 / 2} V^{2} \sqrt{R} K_{+1}^{2}(\gamma a)} \exp \left(-\frac{2 \gamma^{3} R}{3 \beta^{2}}\right),
$$

where $K_{+1}(\gamma a)$ is the modified Hankel function, $\kappa$ is the normalized radial phase constant, $\gamma$ is the normalized radial attenuation constant, $\beta$ is the axial propagated constant, $V$ is the normalized frequency, and $a$ is the radius of the fiber core. These parameters are fixed values when the single-mode optical fiber and the wavelength of incident light are chosen. Therefore, $2 \alpha$ is simplified as follows:

$$
2 \alpha=\frac{A_{1}}{\sqrt{R}} \exp (-B R)
$$

where $A_{1}=\sqrt{\pi} \kappa^{2} / 2 \gamma^{3 / 2} V^{2} K_{+1}^{2}(\gamma a)$ and $B=2 \gamma^{3} / 3 \beta^{2}$.

According to (20) and (21), the coefficient of bending loss in unit length is given as follows:

$$
\begin{aligned}
\alpha_{p} & =4.324 \times 2 \alpha=\frac{4.324 A_{1}}{\sqrt{R}} \exp (-B R) \\
& =\frac{A}{\sqrt{R}} \exp (-B R) .
\end{aligned}
$$

Hence, for the single-model optical fiber with a certain wavelength, the coefficient of bending loss in unit length $\alpha_{p}$ is the function of the bending radius. The bending loss of optical fiber $L_{s}$ with the bending length and the bending radius being $L$ and $R$ is shown as follows:

$$
L_{s}=\frac{A L}{\sqrt{R}} \exp (-B R)=\alpha_{P} L .
$$

\section{Experimental Investigation of the Bending Loss of Optical Fibers under Different Bending Radii}

According to the bending loss mechanism of the optical fiber mentioned in Section 3, the local bending of the optical fiber 


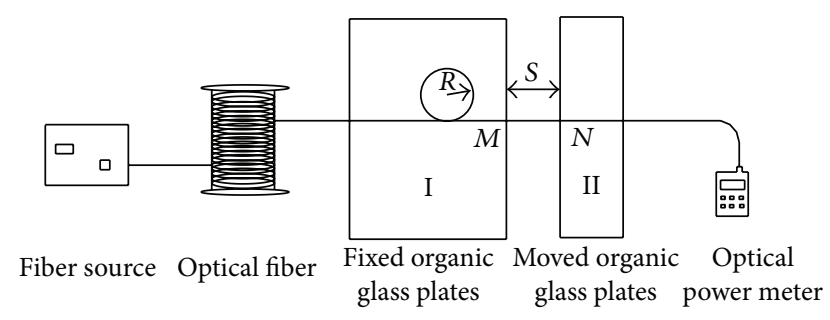

Figure 4: Schematic diagram of the bending loss experiment.

causes the bending loss, and the bending loss is related to the curvature radius $R$, diameter of fiber core $d$, diameter of the cladding $D$, and relative difference of refractive index $\Delta$. Because a single-mode optical fiber is chosen in the experiment, the bending loss is only related to the curvature radius. Therefore, the purpose of this experiment is to study the relationship between the bending radius and the bending loss and to determine the corresponding formula, which provides the monitoring range and sensibility of the singlemode optical fiber.

The main premise behind this experiment is that, by changing the bending radius and measuring the corresponding bending loss by an optical power meter, the nonlinear least square fitting function can be applied to calculate the coefficients $A$ and $B$ in (22).

The instruments used in the experiment include ASE fiber source, a JW3203R Portable Optical Power Meter, SMF28e Single-mode Optical Fiber, a FSM-505 Automatic Singlecore Optical Fiber Fusion Splicer, two organic glass plates, a Vernier Caliper, a CT-30 Optical Fiber Cutter, and a Wire Stripper.

According to (13), for the SMF28e single-mode optical fiber, the critical radius is calculated with the values of $15 \mathrm{~mm}$ and $13 \mathrm{~mm}$, corresponding to the wavelengths of $1550 \mathrm{~nm}$ and $1310 \mathrm{~nm}$. Therefore, the optical fiber was designed to be a circle, and the initial radius is $20 \mathrm{~mm}$ in this work. Then, both sides of the optical fiber were fixed on the two organic glass plates, respectively. One organic glass plate was fixed, while the other one was movable in the horizontal direction. As Figure 4 shows, by measuring the value of $S$ through the Vernier Caliper, the bending radius $R$ was calculated in the following equation:

$$
S=2 \pi\left(R_{\mathrm{ini}}-R\right),
$$

where $R_{\text {ini }}$ is the initial radius with the value of $20 \mathrm{~mm}$.

In addition, the bending length of the optical fiber is described by the following equation:

$$
L=2 \pi R .
$$

Figure 5 illustrates the actual device of the bending loss experiment. The free end of the optical fiber connects to the interference of the optical power meter through the optical fiber connector. During the experiment, the bending radius decreased gradually by intervals of $0.2 \mathrm{~mm}$, until the final bending radius of $2.0 \mathrm{~mm}$. For every change in radius, the bending loss was measured 3 times and the average value was

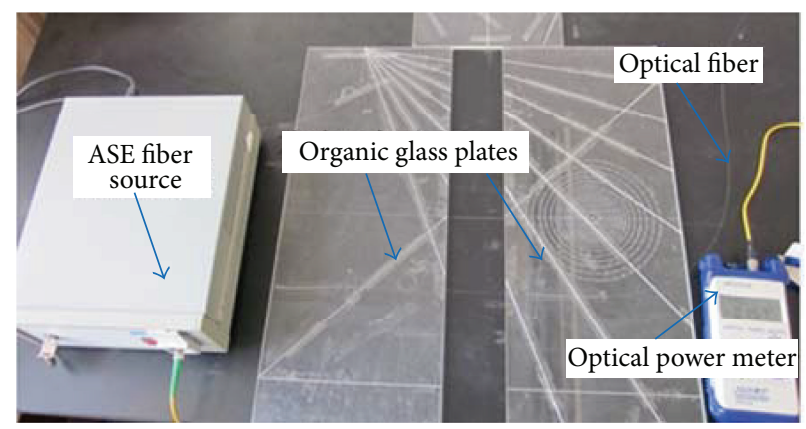

Figure 5: Bending loss experiment device.

TABLE 1: Fitting coefficient, multiple correlation coefficient, and mean square deviation.

\begin{tabular}{lcccc}
\hline Wavelength & $A$ & $B$ & $\begin{array}{c}\text { Multiple correlation } \\
\text { coefficient }\end{array}$ & $\begin{array}{c}\text { Mean square } \\
\text { deviation }\end{array}$ \\
\hline $1310 \mathrm{~nm}$ & 4.1238 & 0.4621 & 0.9465 & 0.0623 \\
$1550 \mathrm{~nm}$ & 10.2684 & 0.3374 & 0.9657 & 0.1135 \\
\hline
\end{tabular}

regarded as the corresponding bending loss. In addition, two wavelengths of the optical fiber with the values of $1310 \mathrm{~nm}$ and $1550 \mathrm{~nm}$ were used to in the detection.

According to the experiment, the bending loss is very slight until the bending radius decreases to the value of $10 \mathrm{~mm}$. As the critical being radii in above experiments are $15 \mathrm{~mm}$ and $13 \mathrm{~mm}$, hence, only the radii of $15 \mathrm{~mm}$ and $13 \mathrm{~mm}$ at range of $10-20 \mathrm{~mm}$ are selected for analysis. In addition, nonlinear least square fitting function was applied to calculate the coefficients $A$ and $B$ in (22). The results are shown in Table 1. In addition, Figure 6 illustrates the relationship between the bending radius and the bending loss for the two cases, both in measured values and fitting values.

As Table 1 shows, the multiple correlation coefficients in the two cases are very large, and it is larger when the wavelength is $1550 \mathrm{~nm}$. By analyzing the relationship between the bending radius and the bending loss per unit length in Figure 6, the following conclusions can be obtained:

(1) For the same wavelength, the bending loss has a negative correlation with the bending radius; that is, the bending loss increases with a decrease in the bending radius. When the bending radius is $15 \mathrm{~mm}$, the curve corresponding to the wavelength of $1550 \mathrm{~nm}$ begins to change dramatically; meanwhile, the curve for the wavelength $1310 \mathrm{~nm}$ begins to change dramatically when the bending radius is $13 \mathrm{~mm}$. Clearly, the point for dramatic changes in curves corresponds to the critical bending radius.

(2) In the above two cases, when the bending radius is bigger than the critical radius, the bending loss is very small; the increased magnitude decreases accordingly with a decrease in the bending radius. Meanwhile, the bending loss increases exponentially with a decrease of the bending radius when the bending radius is smaller than the critical radius. 


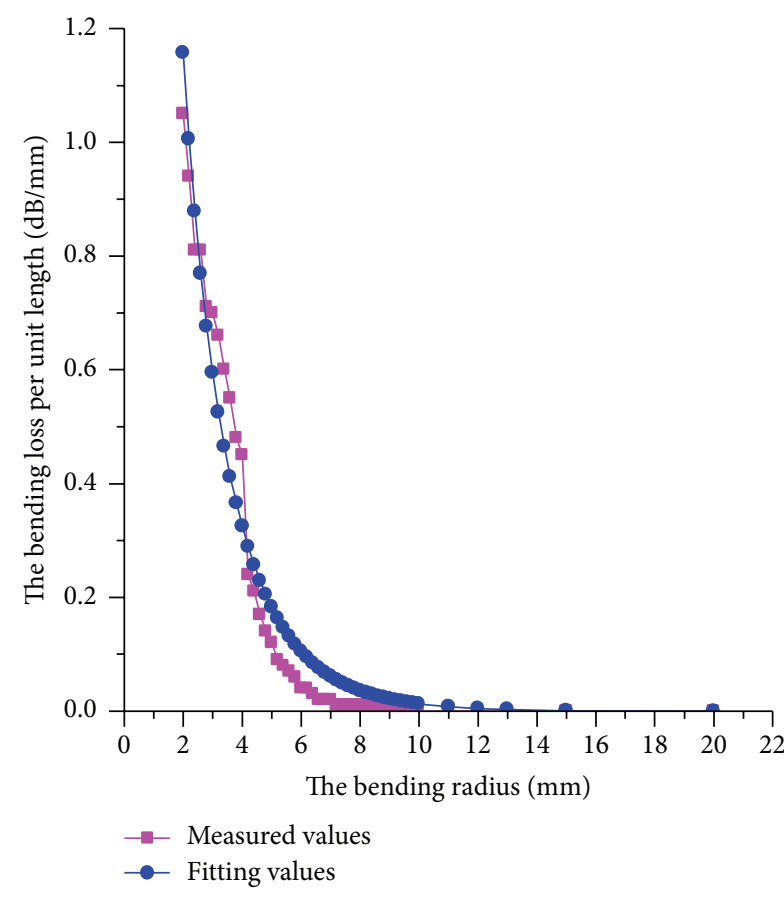

(a)

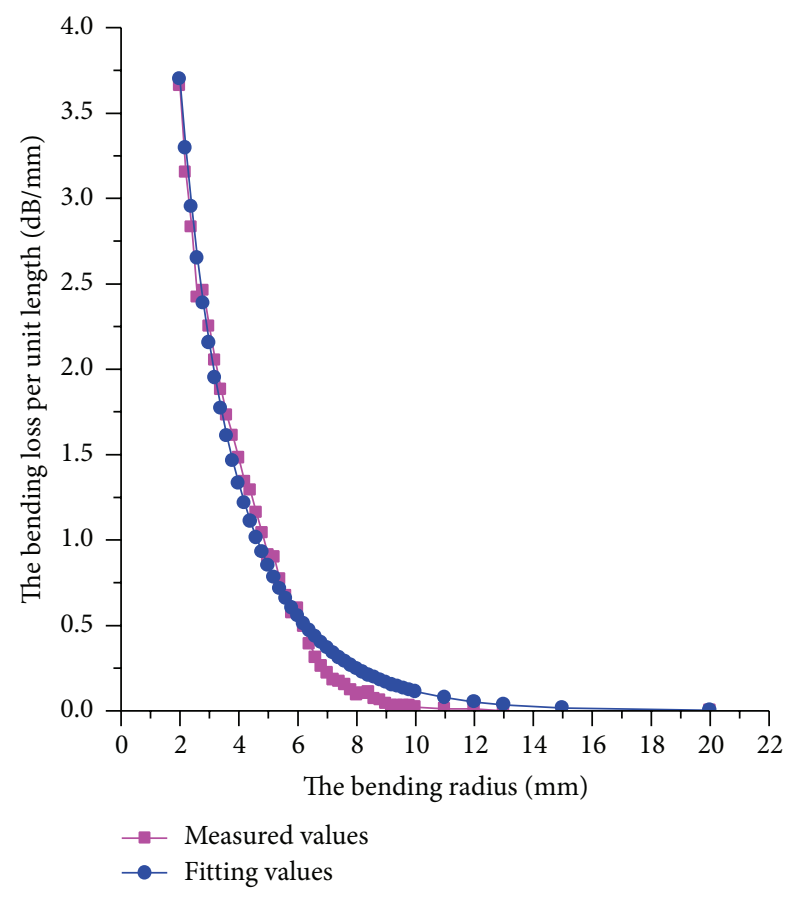

(b)

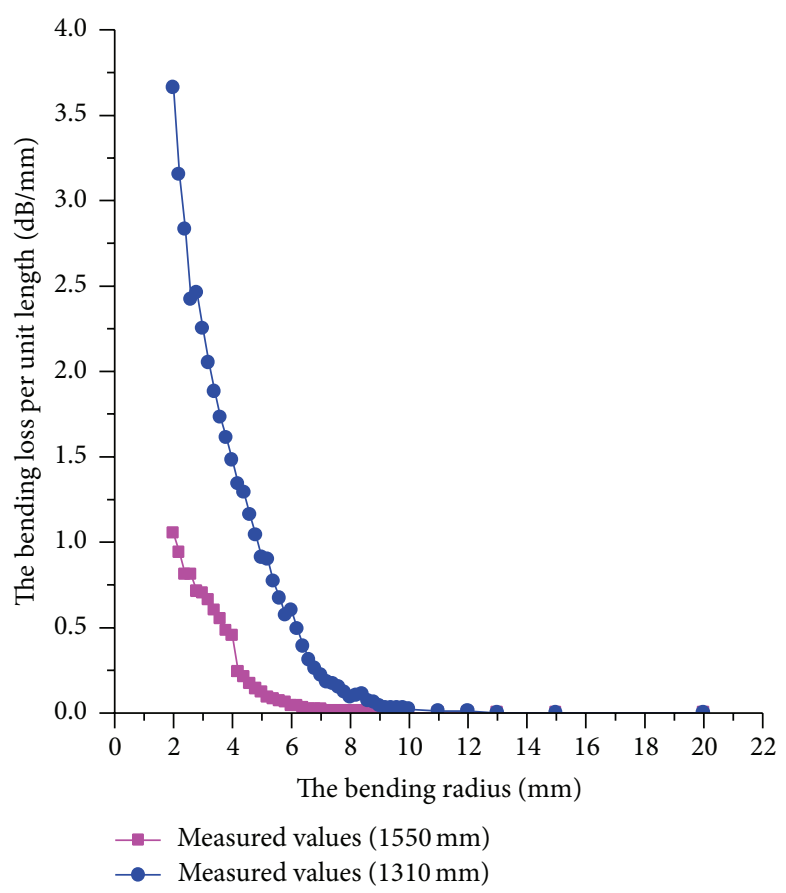

(c)

Figure 6: Relationship between the bending radius and the bending loss per unit length (a) $1310 \mathrm{~nm}$ wavelength, (b) $1550 \mathrm{~nm}$ wavelength, and (c) both waves.

(3) The bending loss for the $1550 \mathrm{~nm}$ wavelength is bigger than that of the $1310 \mathrm{~nm}$ wavelength, which indicates that the longer the wavelength is, the bigger the bending loss will be.
(4) According to the multiple correlation coefficient and the mean square deviation in Table 1, (22) can be successfully applied in calculating the relationship between the bending loss and the bending radius. 


\section{Design and Experimental Investigation of a Butterfly-Type Optical Fiber Sensor}

The bending loss experiment under different bending radii achieved promising results. However, the bending loss increases dramatically when the bending radius is smaller than the critical radius, which demonstrates the relatively low monitoring sensitivity of the outside variation. In addition, the bending loss is quite low when the bending radius is smaller than the critical radius. To enlarge the monitoring range and improve the sensitivity of monitoring, a new type of optical fiber sensor is needed. Based on the previous research $[12,14,15]$, the butterfly-type optical fiber sensor was designed by utilizing the bending loss mechanism of the optical fiber. The sensing performance of the butterfly-type optical fiber sensor was analyzed through a performance experiment.

The instruments used in the experiment included an optical time domain reflectometer (OTDR), SMF28e Singlemode Optical Fiber, organic glass plates, a FSM-505 Automatic Single-core Optical Fiber Fusion Splicer, hard white paper which marked with a scale, plastic capillary, a Vernier Caliper, a CT-30 Optical Fiber Cutter, and a Wire Stripper.

To have a clear understanding of the butterfly-type optical fiber sensor, its manufacturing procedure was described as follows. First, two organic glass plates were positioned to fix the hard white paper. Second, transparent tape was used to attach the plastic capillary to the hard white paper, and the SMF28e Single-mode Optical Fiber was threaded from one end (marked as 1) of the plastic capillary to the other end (marked as 2). Third, the SMF28e Single-mode Optical Fiber was designed to be butterfly-type with a butterfly knot width of $a$. In the next step, the SMF28e Single-mode Optical Fiber was threaded to the plastic capillary again; then, the optical fiber was attached to the end of the plastic capillary marked 1 , and the sticking position was marked as the starting point. Finally, one end of the optical fiber was connected with the interference of the optical time domain reflectometer through the optical fiber connector, while the other end was the free end. During the measuring process, the optical fiber moves gradually from the starting point at intervals of $1 \mathrm{~mm}$ at a time, and the optical time domain reflectometer simultaneously measures the corresponding bending loss.

To obtain the initial perceived radius of the curved butterfly-type optical fiber sensor, the initial width of the butterfly knot is $48 \mathrm{~mm}$ based on the research of Zhu [12]. Each time the optical fiber moved by $1 \mathrm{~mm}$, the bending loss was measured 3 times with the average value as the corresponding bending loss. Figure 7 shows the experimental device in monitoring the relationship between the bending loss and monitoring range of the butterfly-type optical fiber.

The experiment was conducted at a wavelength of $1310 \mathrm{~nm}$, and it began when the width of the butterfly knot was $48 \mathrm{~mm}$. The experimental result shows that the optical time domain reflectometer could barely monitor the optical loss when the width of the butterfly knot is within the range of $45-48 \mathrm{~mm}$. However, the optical loss monitored by the optical time domain reflectometer changes dramatically when the width of the butterfly knot is less than $45 \mathrm{~mm}$. Therefore, only the results after analysis $45 \mathrm{~mm}$ width of the butterfly

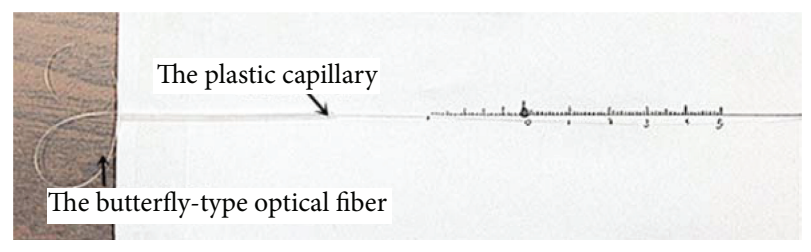

FIGURE 7: Experimental device in monitoring the relationship between the bending loss and the monitoring range of the butterflytype optical fiber.

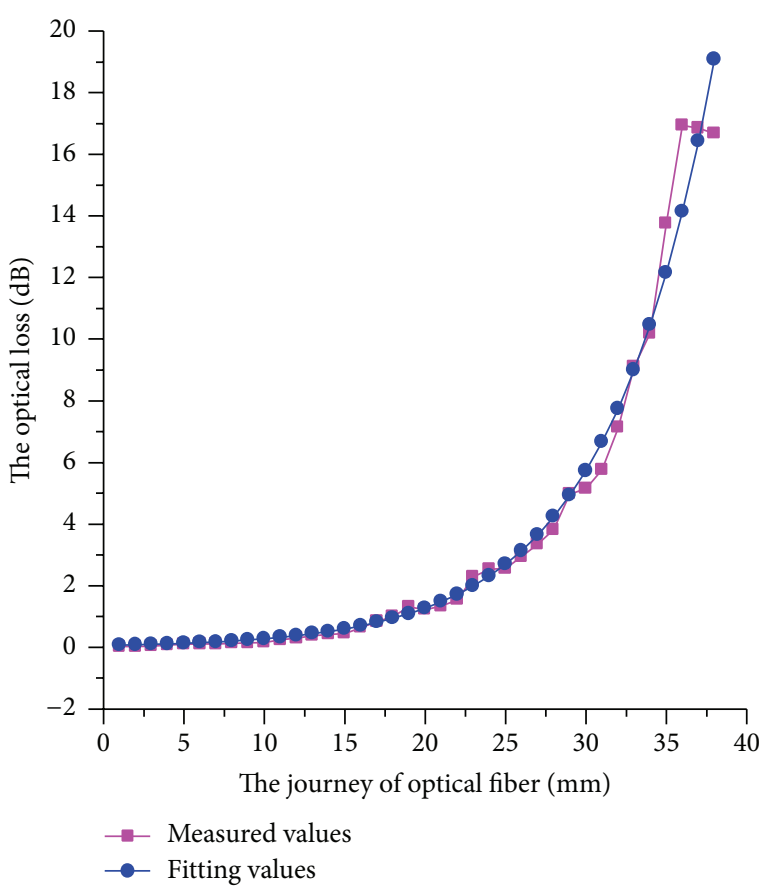

FIGURE 8: Relationship between the optical loss and the journey of the optical fiber.

knot were analyzed. Figure 9 shows the relationship between the optical loss and the journey of optical fiber, and it is evident that the relationship is nonlinear. The bending loss has a positive correlation with the journey of optical fiber; that is, the optical loss grows with the increasing journey of the optical fiber. When the journey of the optical fiber reaches $36 \mathrm{~mm}$, the optical loss does not increase dramatically. Hence, the measurement range of the butterfly-type optical fiber sensor is $0-36 \mathrm{~mm}$. According to the regression analysis of the exponential, power law, the quadric regression, and logistic models, the exponential model has the largest multiple correlation coefficient with the value of 0.9838 , and the mean square deviation is 0.7019 . Therefore, the fitting model is as follows:

$$
\text { Ls }=0.0629 \exp (0.1504 l) \text {, }
$$

where Ls is the optical loss with the unit of $\mathrm{dB}$ and $l$ is the journey of the optical fiber.

According to Figure 8 and (26), the following conclusion can be obtained. 


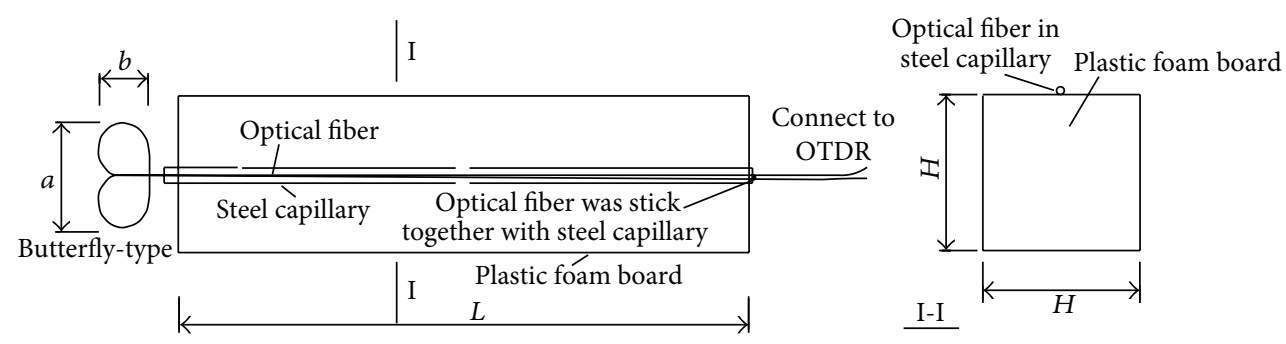

(a)

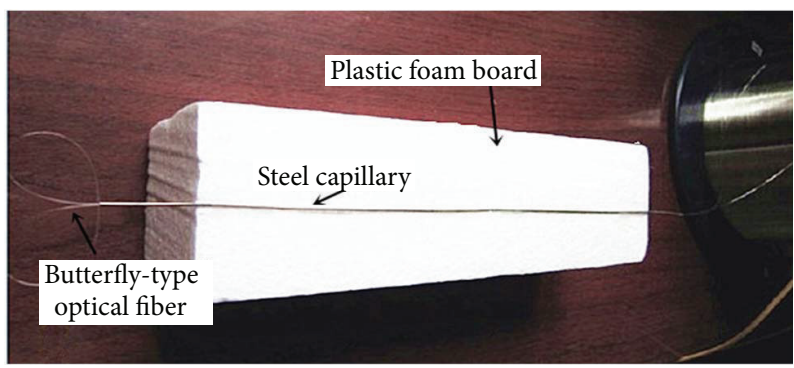

(b)

FIGURE 9: Arrangement of the composite optical fiber monitoring device: (a) diagram and (b) the actual device.

The optical fiber increases with the increasing journey of the optical fiber. When the journey is within the range of $0-$ $30 \mathrm{~mm}$ and the journey increases at intervals of $1 \mathrm{~mm}$, the optical loss increases by a small magnitude. When the journey is within the range of $30-36 \mathrm{~mm}$, the optical loss increases dramatically. In addition, the experiment indicated that the initial measuring accuracy is $1 \mathrm{~mm}$ when the width of the butterfly knot is $45 \mathrm{~mm}$, and the corresponding optical loss is $0.022 \mathrm{~dB}$, which demonstrates a higher initial measuring accuracy of the optical fiber sensor. At the measurement range of 0-36 mm, the butterfly-type optical fiber sensor has a larger monitoring journey. Furthermore, the fitting precision of (26) is large enough to prove the success of the equation in representing the relationship between the optical loss and the journey of the optical fiber.

\section{Study of the Composite Optical Fiber Sensor Monitoring Device in Monitoring the Differential Settlement in the Earth-Rock Junction}

The composite optical monitoring device for monitoring the differential settlement in the earth-rock junction proposed in this work aimed for a higher initial accuracy and larger measuring range. The components of the monitoring device include the optical time domain reflectometer (OTDR), plastic foam board, steel capillary with an external diameter of $3 \mathrm{~mm}$ and inner diameter of $2.5 \mathrm{~mm}$, single-mode optical fiber, and the epoxy resin adhesive.

6.1. Working Principles of the Composite Optical Fiber Sensor Monitoring Device. As Figure 9 shows, the steel capillary was cut off at the loading point, and it was attached to the plastic foam board with epoxy resin adhesive. When the section I-I was under the concentrated or the distributed load, the optical loss could be measured by the optical time domain reflectometer. Certainly, the plastic foam board would have deformation when subjected to load. Because the steel capillary was stick together with the plastic foam board, the steel capillary and the optical fiber in the steel capillary would have cooperative deformation. As one end of the optical fiber was connected to the steel capillary, the size of the butterfly knot $a$ and butterfly knot $b$ would decrease. The optical fiber at the end of the butterfly knot would have large bending loss as the deformation increases.

Moreover, when the plastic foam board sustains lower pressure, the bending loss appears at the end of the butterfly knot. The optical fiber could be extended for few centimeters from the initial deformation to breaks. Therefore, high initial measuring sensibility and a large measurement range were achieved.

\subsection{Flexural Behavior of the Composite Optical Fiber Sensor} Monitoring Device. As Figure 9 shows, as the substrate of the monitoring device, the plastic foam board was assumed to be an elastic material that conforms to Hooke's Law. Because the bending model of the monitoring device is similar to that of a beam, the composite monitoring device was analyzed by treating it as the beam structure. Therefore, according to the theory of beam deformation, the lower part of a beam is subject to tension while the upper part of a beam is subject to compression.

If the optical fiber was located at the lower surface of the model, the optical fiber would be in tension, and, as a result, the optical fiber would extend and the optical loss of optical fiber at the end of the butterfly knot would then increase. If the optical fiber was located at the neutral axis, the optical 


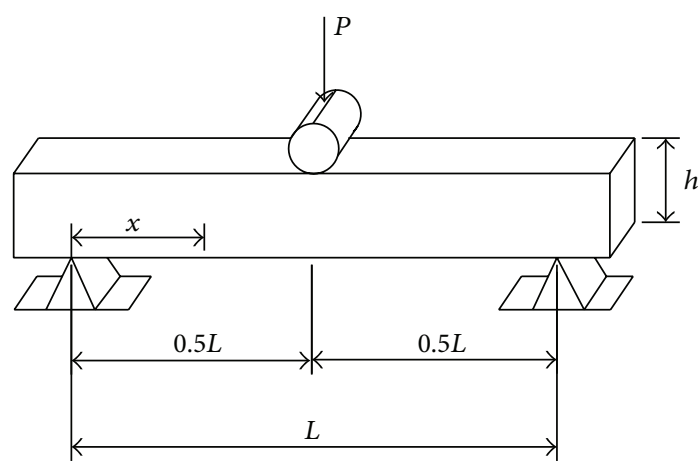

FIGURE 10: Bending model of the composite monitoring device.

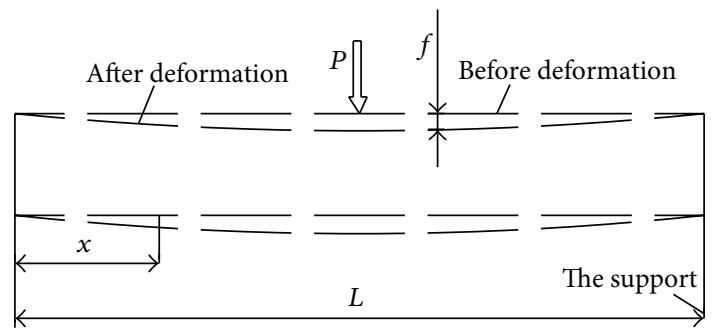

FIGURE 11: Composite monitoring device in the linear stage.

fiber would not extend or shorten and no optical loss would exist. If the optical fiber was located at the upper surface of the model, the optical fiber would compress and no optical loss would occur. As the optical loss should be measured, we arranged the optical fiber at the lower surface of the model.

The elastic plastic foam board has linear elastic deformation until the cross section was broken. Therefore, the analysis of the flexural behavior includes a linear stage and break stage. Figure 10 shows the bending model of the composite monitoring device.

6.2.1. Linear Stage. As Figure 11 shows, if the midspan of the device was subjected to a load for a magnitude of $P$, the deflection at the midspan could be calculated as follows according to structural mechanics:

$$
f=\frac{P L^{3}}{96 E I} .
$$

Therefore, the magnitude of the load $P$ could be obtained:

$$
P=\frac{96 E I f}{L^{3}} .
$$

The moment at the cross section with the distance of $x$ from the left support is described as follows:

$$
M=\frac{P x}{2} .
$$

Then, the maximum tensile stress in the lower surface is calculated:

$$
\sigma=\frac{M}{I} y=\frac{M}{I} \frac{h}{2} .
$$

Therefore the strain at the cross section is given in the following equation:

$$
\varepsilon_{x}=\frac{\sigma}{E}=\frac{M}{I} \frac{h}{2 E}=\frac{h}{2 E I} \frac{x}{2} \frac{96 E I f}{L^{3}}=\frac{24 f h x}{L^{3}} .
$$

Then, the extended length of optical fiber is described as follows:

$$
\Delta L=2 \int_{0}^{L / 2} \varepsilon_{x} d x=\frac{6 h}{L} f
$$

where $\Delta L$ is the extended length of the optical fiber, $L$ is the length of the monitoring device, and $h$ is the height of the monitoring device.

6.2.2. Break Stage. The extended length is related to the deflection $f$, height $h$, and length $L$. By analyzing the geometrical relationship in Figure 12, the following equation is obtained:

$$
\Delta L=\frac{2 h\left[h L-(h-f) \sqrt{L^{2}-2 f h+f^{2}}\right]}{L^{2}+(h-f)^{2}},
$$

where $\Delta L$ is the extended length of the optical fiber, $L$ is the length of the monitoring device, and $h$ is the height of the monitoring device.

6.3. Bending Experiment of the Composite Optical Fiber Sensor Monitoring Device. By analyzing the flexural behavior of the composite optical fiber sensor monitoring device, we obtained the working principle. However, the feasibility of the composite monitoring device was preliminary verified by conducting the bending experiment of the composite optical fiber sensor monitoring device.

The instruments used in the experiment included a universal testing machine, plastic foam board, steel capillary, an optical time domain reflectometer (OTDR), an SMF28e Single-mode Optical Fiber, an FSM-505 Automatic Singlecore Optical Fiber Fusion Splicer, a Vernier Caliper, a CT30 Optical Fiber Cutter, a Wire Stripper, the epoxy resin adhesive, and a weld heat-shrinkable tube.

Figure 13 shows the bending experiment of the composite monitoring device, where the substrate of the composite optical fiber sensor monitoring device is the plastic foam board $(300 \mathrm{~mm} \times 75 \mathrm{~mm} \times 60 \mathrm{~mm}$ ) and the span is $150 \mathrm{~mm}$. The steel capillary was attached to the plastic foam board, which was broken at the midspan. The optical fiber in the steel capillary was attached to one end of the steel capillary, and the optical fiber at the other end of the steel capillary was designed to be butterfly-type with the width of $45 \mathrm{~mm}$. The end of the bowknot was marked as the starting position. One end of the optical fiber connects to the interference of the optical power meter through the optical fiber connector, and the optical loss was measured by the optical time domain reflectometer. Moreover, the vertical displacement at the loading point was measured by ruler.

The relationship between the optical loss and the vertical displacement at the loading point can be determined 


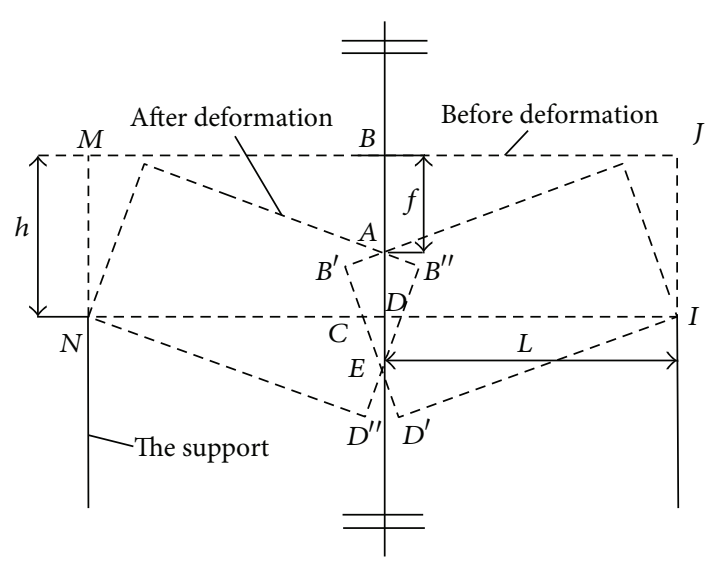

(a)

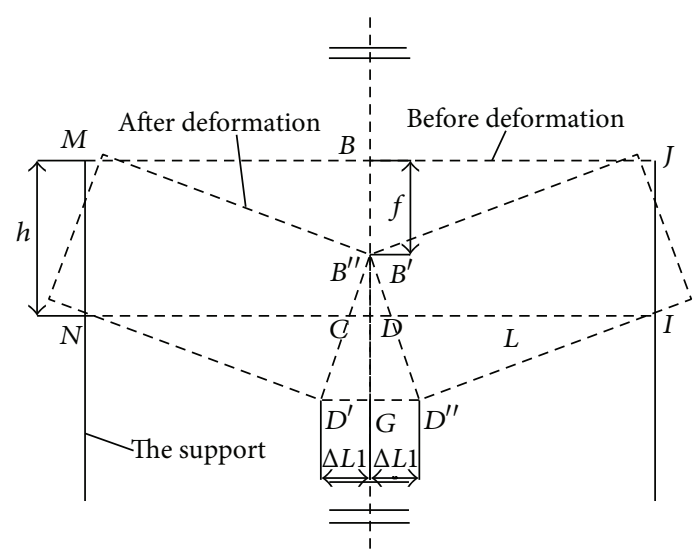

(b)

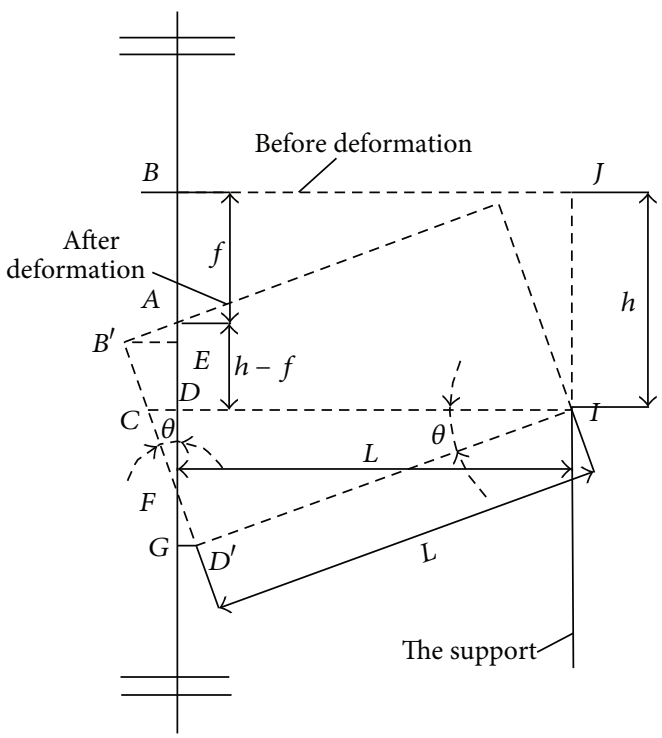

(c)

FIGURE 12: Composite monitoring device in the break stage: (a) the upper surface retracts, (b) the actual situation, and (c) enlarged diagram of the right side.

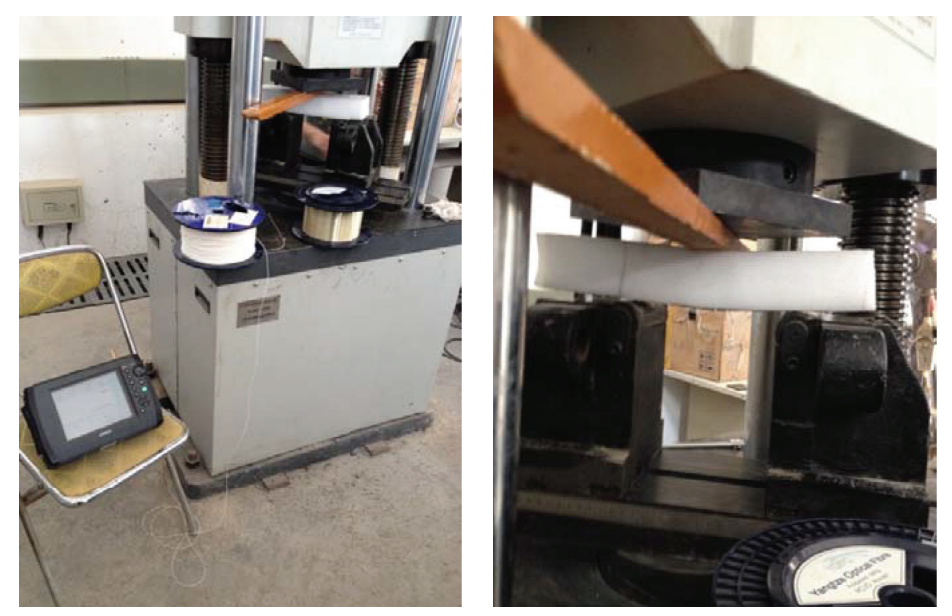

FIGURE 13: Bending experiment of the composite monitoring device. 


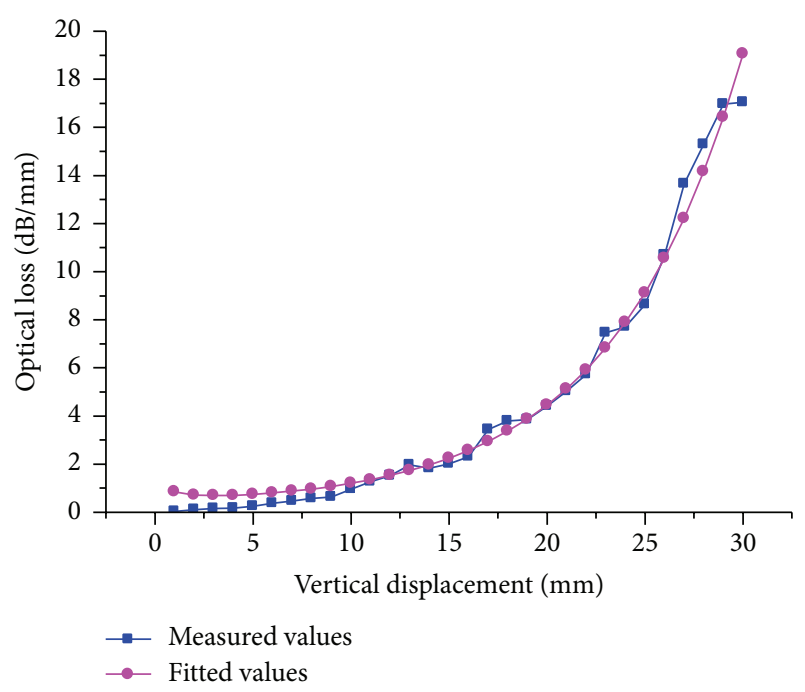

FIGURE 14: Relationship between the optical loss and the vertical displacement of the loading point.

TABLE 2: Fitting coefficient, multiple correlation coefficient, and mean square deviation.

\begin{tabular}{lcccc}
\hline Wavelength & $A$ & $B$ & $\begin{array}{c}\text { Multiple correlation } \\
\text { coefficient }\end{array}$ & $\begin{array}{c}\text { Mean square } \\
\text { deviation }\end{array}$ \\
\hline $1310 \mathrm{~nm}$ & 0.727 & -0.136 & 0.972 & 0.622 \\
\hline
\end{tabular}

through the experiment, as shown in Figure 14. In addition, a nonlinear least square fitting function was applied to fit the relationship, and the results are shown in Table 2.

By analyzing the experiment, we conclude that the optical loss has a positive correlation with the vertical displacement; that is, the optical loss increases correspondingly with increasing vertical displacement. Considering the large multiple correlation coefficients in Table 2, (22) can be applied in establishing the relationship. The good result indicates that the composite optical fiber sensor monitoring device could undertake a larger range of measurement.

\subsection{Experiment of the Composite Optical Fiber Sensor Mon-} itoring Device in Monitoring the Differential Settlement of Soils. Based on the composite optical fiber sensor monitoring device mentioned above, the following model was applied in monitoring the differential displacement of the earth-rock junction to verify the feasibility of the composite monitoring device. The instruments used in the experiment included a hydraulic jack specified 5 tons, plastic foam board, steel capillary, an optical time domain reflectometer (OTDR), an SMF28e Single-mode Optical Fiber, an FSM-505 Automatic Single-core Optical Fiber Fusion Splicer, a Vernier Caliper, a CT-30 Optical Fiber Cutter, a Wire Stripper, epoxy resin adhesive, a weld heat-shrinkable tube, soil, and the wooden box $(260 \mathrm{~mm} \times 260 \mathrm{~mm} \times 360 \mathrm{~mm})$.

The plastic foam board was located at the position shown in Figure 15. The steel capillary was attached to the plastic foam board. Then, the butterfly-type optical fiber with the butterfly knot (width: $45 \mathrm{~mm}$ ) was positioned in the steel capillary, while the other end of the optical fiber was attached to the steel capillary. The middle of the steel capillary was cut with a kerf. The wooden box was then filled with soil to the top of the wooden box, and the wooden box was covered with boards in the next phase so that the soil would be subjected to uniform stress. The hydraulic jack was uploaded to the structure, and then the settlement of the soils was measured by the Vernier Caliper. One end of the optical fiber connects to the interference of the optical power meter through the optical fiber connector, and the optical loss was measured by the optical time domain reflectometer. In addition, the experiment was conducted under a wavelength of $1310 \mathrm{~nm}$.

However, as the soil in the wooden box is not dense, the optical loss was not measured until the displacement reached $17 \mathrm{~mm}$. Figure 16 illustrates the exponential relationship between the optical loss and the vertical displacement of the loading point. We concluded that the composite monitoring device could monitor the vertical displacement.

\section{Conclusions}

This work aimed at applying optical fiber sensor to monitor the differential settlement of an earth-rock junction, combined with the theoretical analysis of bending loss mechanism of optical fiber and a series of experiments. A monitoring device and monitoring methods based on the theory of bending loss were studied, which provided technical support for the optical fiber sensor applied in monitoring the differential settlement of the earth-rock junction. The main content of this paper is as follows:

(1) Based on the bending loss mechanism of optical fibers, an experiment on the bending loss under different bending radii was developed and analyzed. By analyzing the relationship between the optical loss and bending radius, we found that the single-mode optical fiber has weaknesses in its small monitoring range and low monitoring sensibility.

(2) Based on the theory of bending loss of optical fibers, a butterfly-type optical fiber sensor was designed, which has a relatively larger monitoring range and higher monitoring sensibility. The relationship between the optical loss and the journey of optical fiber was then established. On this basis, a composite optical fiber sensor monitoring device was designed to monitor the differential settlement of soils. By analyzing the flexural behavior of the composite optical fiber sensor monitoring device, the journey of the optical fiber was determined to have a linear relationship with the optical loss before the device is broken; it is a nonlinear relationship before the device is broken. Through the bending experiment and the experiment of the composite monitoring device in monitoring the differential settlement of soils, a larger monitoring range and high monitoring sensibility are achieved, which provide technical support for the composite monitoring device applied in monitoring the differential settlement of the earth-rock junction. 


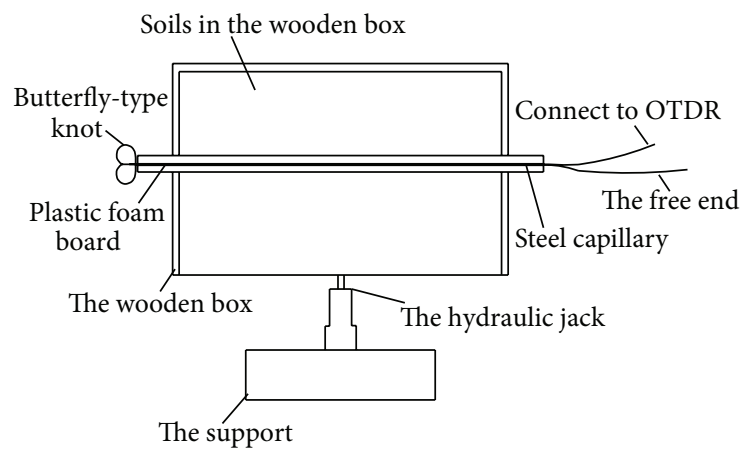

(a)
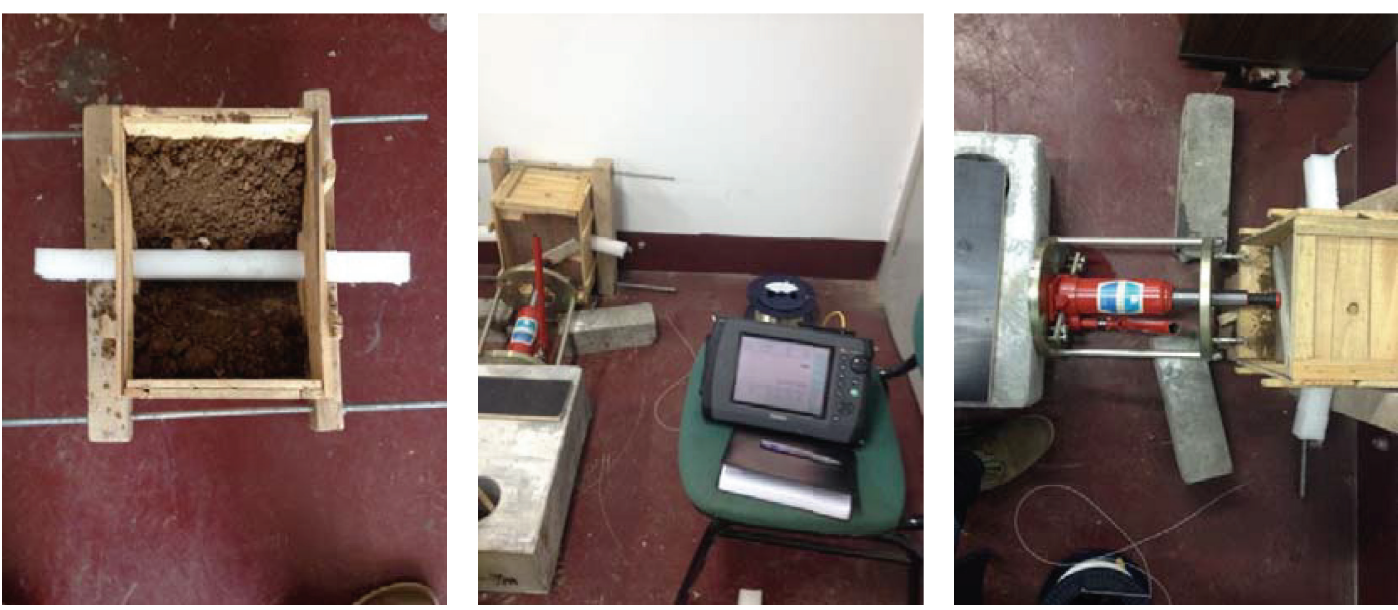

(b)

FIGURE 15: Composite device experiment in monitoring the differential settlement of soils: (a) diagram and (b) the actual device.

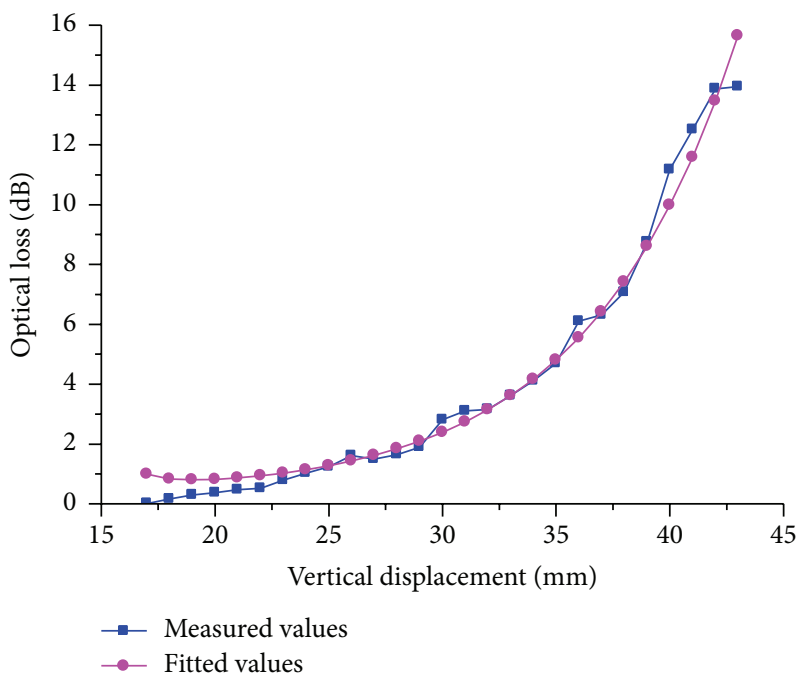

FIGURE 16: Relationship between the optical loss and the vertical displacement of the loading point.

\section{Conflict of Interests}

The authors declare that there is no conflict of interests regarding the publication of this paper.

\section{Acknowledgments}

This work was supported by the National Natural Science Foundation of China (Grant nos. 51279052, 51139001, 2014513311, 51409205, and 81279052), China State Key Laboratory of Hydrology-Water Resources and Hydraulic Engineering (Grant no. 20145028312), Jiangsu Province “333 HighLevel Personnel Training Project” (Grant nos. 2016-B1307101, 2017-B08037), Open Fund of Key Laboratory of Earth-Rock Dam Failure Mechanism and Safety Control Techniques, Ministry of Water Resources (Grant no. YK914022).

\section{References}

[1] H. Su, J. Hu, and M. Yang, "Dam seepage monitoring based on distributed optical fiber temperature system," IEEE Sensors Journal, vol. 15, no. 1, pp. 9-13, 2015.

[2] T. Tianguo, C. Chunhua, and L. Haowu, "Application of distributed optical fiber sensors into crack monitoring of dam's foundation," Chinese Journal of Sensors and Actuators, vol. 20, no. 10, pp. 2357-2360, 2007.

[3] Y. Wu, H. Su, and P. Gao, "Integrated study on key basic problems of fiber optic monitoring cracks in concrete large dams," Journal of Hydroelectric Engineering, vol. 26, no. 4, pp. 120-123, 2007. 
[4] P. Zhu, Y. B. Leng, Y. Zhou, and G. L. Jiang, "Safety inspection strategy for earth embankment dams using fully distributed sensing," Procedia Engineering, vol. 8, pp. 520-526, 2011.

[5] B. Shi, X.-J. Xu, D. Wang et al., "Study on BOTDR-based distributed optical fiber strain measurement for tunnel health diagnosis," Chinese Journal of Rock Mechanics and Engineering, vol. 24, no. 15, pp. 2622-2628, 2005.

[6] H. Mohamad, K. Soga, P. J. Bennett, R. J. Mair, and C. S. Lim, "Monitoring twin tunnel interaction using distributed optical fiber strain measurements," Journal of Geotechnical and Geoenvironmental Engineering, vol. 138, no. 8, pp. 957-967, 2012.

[7] H. H. Zhu, B. Shi, J. F. Yan, J. Zhang, and J. Wang, "Investigation of the evolutionary process of a reinforced model slope using a fiber-optic monitoring network," Engineering Geology, vol. 186, pp. 34-43, 2015.

[8] A. Hessainia, S. El-Akrmi, H. Triki, and A. El-akrmi, "Analysis of fiber Bragg grating with exponential-linear and parabolic taper profiles for dispersion slope compensation in optical fiber links," Optik-International Journal for Light and Electron Optics, vol. 125, no. 17, pp. 4642-4645, 2014.

[9] J. Hu, Z. Qian, and L. Chen, "Fracture behavior of epoxy asphalt pavement on steel bridges based on optical fiber sensing technology and numerical simulation," Journal of Wuhan University of Technology-Mater, vol. 29, no. 4, pp. 858-862, 2014.

[10] H. J. Yoon, K. Y. Song, H. M. Kim et al., "Strain monitoring of composite steel girder bridge using distributed optical fibre sensor system," Procedia Engineering, vol. 10, pp. 2544-2547, 2011.

[11] Y. Miao, The Principle and Application of Fiber Optics, Tsinghua University Press, Beijing, China, 2010.

[12] Z. Zhu, Application and Study on Combined Fiber Optical Sensor for Slope Monitoring, Chongqing University, 2011.

[13] D. Marcuse, "Curvature loss formula for optical fibers," Journal of the Optical Society of America, vol. 66, no. 3, pp. 216-220, 1976.

[14] B. Liu, The Research of the Feasibility Aquiring the Information of Landslide Mechanics Based on Complex Optical Devices, Chongqing University, 2011.

[15] L. Hu, Experimental study on the performance of combined optical fiber sensing for landslide monitoring [M.S. thesis], Chongqing University, Chongqing, China, 2012. 

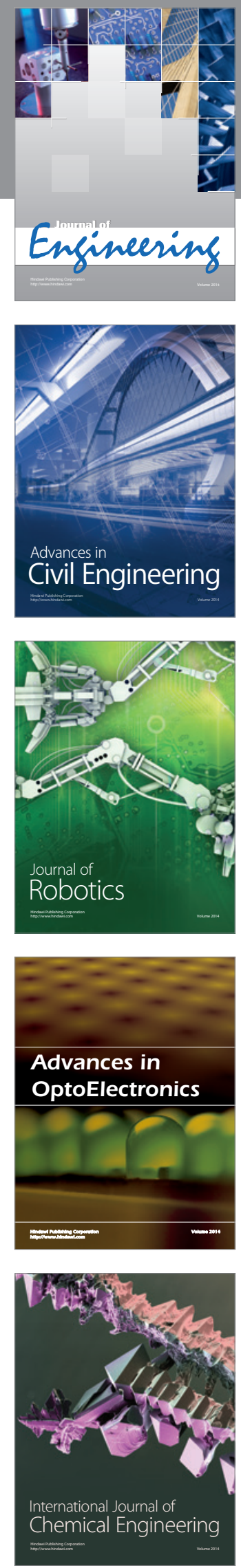

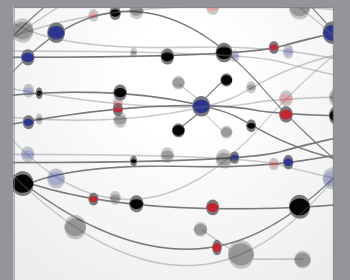

The Scientific World Journal
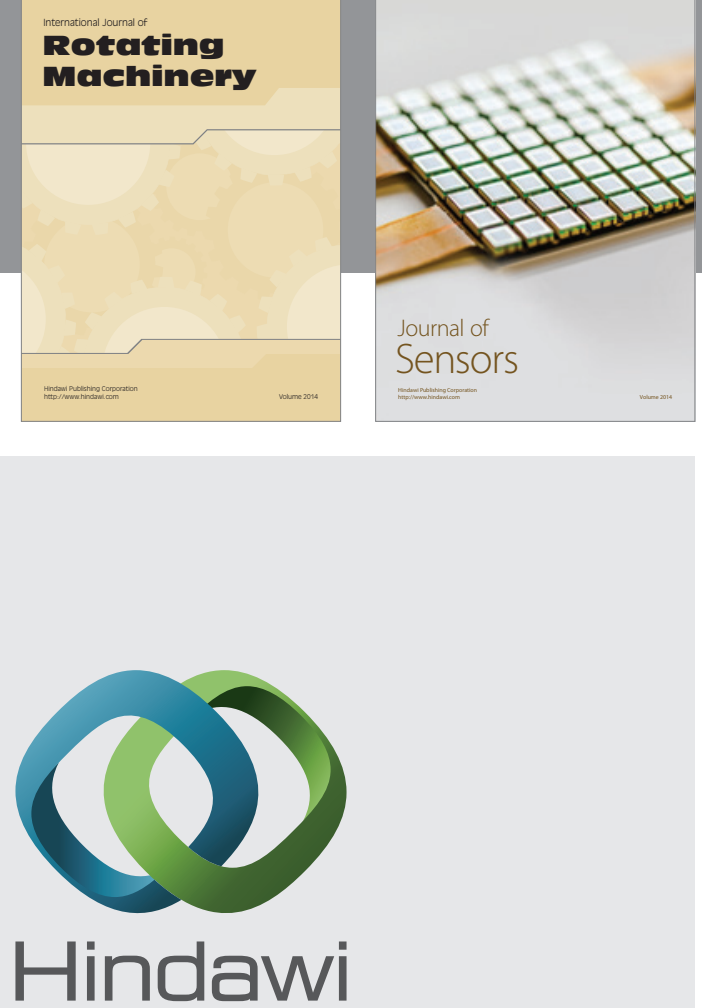

Submit your manuscripts at http://www.hindawi.com
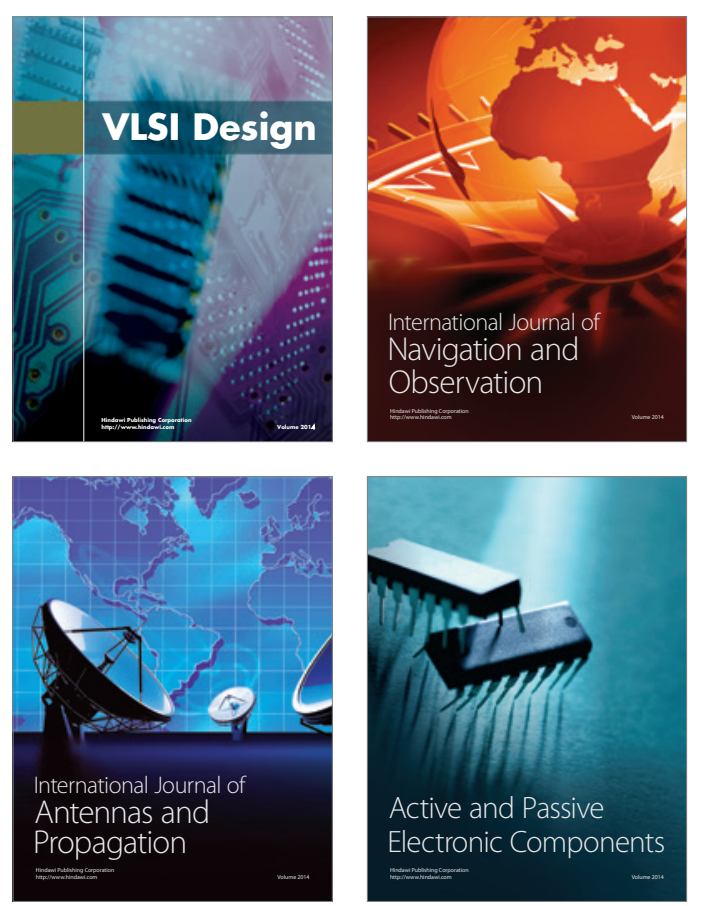
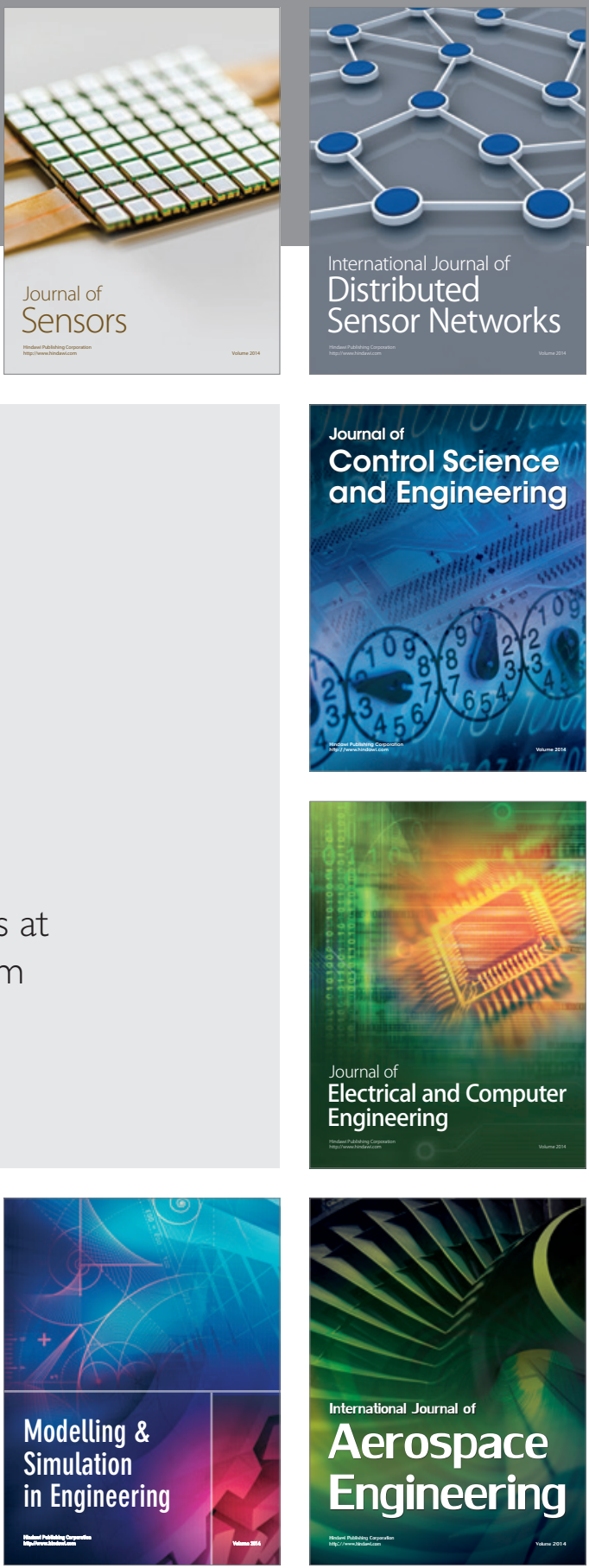

Journal of

Control Science

and Engineering
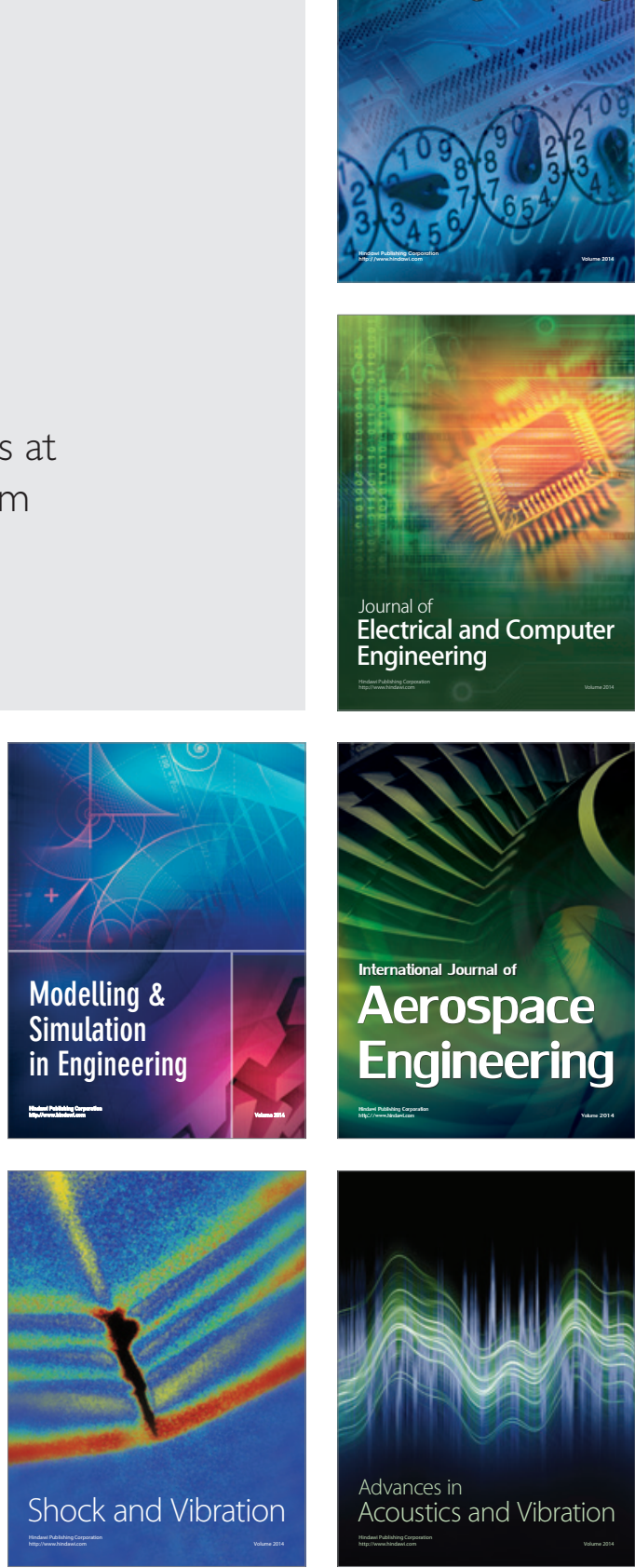